This text was published in Leitner, Ulrich (Hg.): Corpus Intra Muros. Eine Kulturgeschichte räumlich gebildeter Körper. transcript Bielefeld 2017. It is posted here by permission of transcript Verlag for personal use only, not for redistribution.

https://doi.org/10.14361/9783839431481-014

\title{
Gemeinsam im Seminar
}

\author{
Die körperlich-räumliche Herstellung eines
}

Interaktionsereignisses in der Universität

CORDULA SCHWARZE

Der vorliegende Beitrag ordnet sich den Überlegungen zu gebautem Raum zu, in die Reihe von Kloster, Schule und Heim kann auch die Universität gestellt werden. Wie die genannten Räume auch, ist die Universität stark institutionalisiert, hoch spezialisiert durch ihre Aufgaben und funktional dreifach - Forschungs-, Bildungsbzw. Lehr- sowie Verwaltungsfunktion - charakterisierbar. Ein wesentliches Merkmal der Universität, das auf die beiden ersten Funktionen rekurriert, ist der teils fachspezifisch ausdifferenzierte Umgang mit Wissen und Können. Dabei kann die Universität eine lange Tradition für sich reklamieren, die in Europa mit der Universität Bologna im 11. Jahrhundert beginnt. Die Benennung einer Universität durch die Zuordnung zur Stadt, in der sie sich befindet und mit deren Entwicklung sie selbst eng verbunden ist, wie beispielsweise an der Bezeichnung mancher Städte als Universitätsstadt sichtbar ist, weist auf eine enge Ortsbindung der darin stattfindenden Interaktion hin. ${ }^{1}$

1 Ein Beispiel dafür ist die von der Stadt Wien als ein städtisches Ereignis gefeierte Wiedereröffnung des Audimax der Universität Wien im Jahr 2006. Die darin zum Ausdruck kommende Auffassung von Universität und Öffentlichkeit zeigt Zugehörigkeit und Verantwortlichkeit, wobei das Audimax als Ort angesehen wird, an dem sich die Universität der Öffentlichkeit stellt. Die im Audimax zur feierlichen Wiedereröffnung gehaltene Ringvorlesung fasst den Ort über seine Funktion: »Ein solcher Saal ist nichts ohne die Vitalität jener Form, die spezifisch ist für die Institution Universität: die Vorlesung« und konzipiert diese als Schnittstelle zwischen akademischem und öffentlichem Leben, also zwischen innen und außen. Vgl. Arno Dusini / Lydia Miklautsch, Vorwort, in: Vorlesung, hg. von Arno Dusini / Lydia Miklautsch., Göttingen 2007, 7-8. Vgl. zur Verbindung der 
Neben dieser statischen lokalen Verortung hat der Zusammenhang von Universität und Raum aber auch eine dynamische Komponente. Die Ausgangsfrage des Buches sowie des zugrundeliegenden Projekts - Wie werden Räume gebildet und wie bilden sie den Menschen? - ist selbstverständlich auch auf die Universität beziehbar. Dass sich im gebauten Raum der Universität Körper bewegen, also Menschen unterrichten, forschen, lehren, lernen und miteinander sprechen, ist unstrittig. Räume, vor allem gebaute Räume, speichern, wie Ulrich Leitner schreibt, ${ }^{2}$ die Präsenz von Menschen. Dabei sind Körper und Raum wechselseitig aufeinander bezogen: Körper ist ohne Raum nicht zu denken und umgekehrt, erst der Körper macht Raumerfahrung möglich. ${ }^{3}$ Bei der Herstellung von situierter Interaktion in einem besonderen Raum, wofür sich der vorliegende Beitrag interessiert, stehen die Interaktionsbeteiligten in einem raum-zeitlichen Bezugssystem. ${ }^{4}$ Körper und Raum sind darin eng verschränkt und diese Verschränkung muss durch Wahrnehmbarkeit auffallen:

»In einer konkreten Situation kann Raum als interaktive Ressource nur durch die in der Körperlichkeit der Beteiligten verankerten Wahrnehmungs- und Ausdrucksressourcen und nur durch eine aktive Bezugnahme auf Aspekte der gegebenen physikalischen Räumlichkeit in Form eines Blicks, der Handhabung eines Gegenstandes oder durch die Begehung einer freien Fläche realisiert werden. $\ll^{5}$

Corpus intra muros heißt daher für den vorliegenden Beitrag: Menschen in ihrer leiblichen Verfasstheit, funktional differenziert als Studierende und Lehrende, befinden und bewegen sich an einem durch spezifische Zugangsbedingungen ausgezeichneten Ort, der Universität, und interagieren miteinander in ortstypischen Situationen und Rahmungen, also denen des Lehrens und Lernens im Seminar. Die spe-

Universitäten mit ihren Städten: Gastone Ave, University Cities. A Strategic Resource of Small and Medium-Sized Cities in Europe, in: Cities as Multiple Landscapes. Investigating the Sister Cities Innsbruck and New Orleans (Interdisciplinary Urban Studies 21), hg. von Christina Antenhofer / Günter Bischof / Robert Dupont / Ulrich Leitner, Frankfurt am Main / New York 2016, 61-82.

2 Ulrich Leitner in der Einleitung zu diesem Band.

3 Markus Schroer, Räume, Orte, Grenzen, Frankfurt am Main, 4. Aufl., 2012; Heiko Hausendorf / Lorenza Mondada / Reinhold Schmitt, Raum als interaktive Ressource. Eine Explikation, in: Raum als interaktive Ressource. hg. von Heiko Hausendorf / Lorenza Mondada / Reinhold Schmitt, Tübingen 2012, 7-36.

4 Arnulf Deppermann / Reinhold Schmitt, Koordination. Zur Begründung eines neuen Forschungsgegenstandes, in: Koordination. Analysen zur multimodalen Interaktion, hg. von Arnulf Deppermann / Reinhold Schmitt, Tübingen 2007, 15-54, 29.

5 Reinhold Schmitt, Körperlich-räumliche Aspekte der Interaktion, Tübingen 2013, 52. 
zifische Ordnung von Körper und Raum bei der Herstellung einer Seminarsituation ist rekonstruierbar. Das wird im vorliegenden Beitrag aus der Perspektive der linguistischen multimodalen Interaktionsanalyse präsentiert. Dabei werden insbesondere das Konzept der Interaktionsarchitektur ${ }^{6}$ und das der Benutzbarkeitshinweise ${ }^{7}$ angewendet, um zu zeigen, wie Interagierende in spezifischer Weise mit räumlichen und mobiliaren Benutzbarkeitshinweisen umgehen, um eine lehr-/lernorientierte Praxis zu konstituieren. Nach rahmengebenden Erläuterungen zu interaktionslinguistischen Perspektiven auf Raum sowie zum Seminar wird exemplarisch an drei Fällen gezeigt, wie eine räumlich zunächst gegebene Seminarsituation als Interaktionssituation von den Beteiligten körperlich und räumlich hergestellt wird. Die empirische Basis hierfür bildet das Korpus Auswertungsgespräche, ein systematisch erhobenes Videokorpus von Lehr-/Lerninteraktionen in universitären Seminaren einer österreichischen Germanistik. ${ }^{8}$ Die Kombination von Standbild- und Interaktionsanalysen, repräsentiert durch Transkripte, ermöglicht, die »Ordnung des Nebeneinanders «, die den Raum kennzeichnet, und die »Ordnung des Nacheinanders «, ${ }^{9}$ die die Zeit kennzeichnet, aufeinander zu beziehen.

\section{Interaktionsanalytische Perspektiven auf Raum}

Ein Zugang zu Raum, der dessen soziale Konstruiertheit durch individuelles und soziales Handeln betont, stellt für die Frage der räumlich-körperlichen Konstellation im Seminar den passenden theoretischen Rahmen dar. Ein relationaler Raumbegriff betont »die kreativen Möglichkeiten und Chancen der Akteure bei der Konstituierung, dem Aufbau und der Gestaltung von Räumen ${ }^{10}{ }^{10}$ und fokussiert, dass Raum entsteht, gebaut und genutzt wird durch die Verknüpfung mit Objekten und

6 Heiko Hausendorf / Reinhold Schmitt, Interaktionsarchitektur und Sozialtopografie: Umrisse einer raumlinguistischen Programmatik. Arbeitspapiere des UFSP Sprache und Raum SpuR 01, Zürich 2013.

7 Heiko Hausendorf, Über Tische und Bänke. Eine Fallstudie zur interaktiven Aneignung mobiliarer Benutzbarkeitshinweise an der Universität, in: Raum als interaktive Ressource, hg. von Heiko Hausendorf / Lorenza Mondada / Reinhold Schmitt, Tübingen 2012, 139-186.

8 Eine ausführlichere Beschreibung des Korpus findet sich im Abschnitt Datenbasis sowie in: Cordula Schwarze, Das Auswertungsgespräch in der kompetenzorientierten Hochschullehre. Eine interaktionsanalytische Untersuchung, in: Sprechwissenschaft. Bestand, Prognose, Perspektive, hg. von Ines Bose / Baldur Neuber, Frankfurt am Main / New York 2014, 137-147.

9 Begriffe nach Schroer, Räume (wie Anm. 3).

10 Schroer, Räume (wie Anm. 3) 175. 
mit Handlungen, er wird so zu einem Ort (topos). ${ }^{11}$ Diese handlungstheoretische Ausrichtung zeichnet auch das relationale Raumkonzept der multimodalen Interaktionsanalyse aus.

In der Perspektive der linguistischen multimodalen Interaktionsanalyse ${ }^{12}$ sind Raum und Räumlichkeit nur als interaktive Ressource (interactive achievement) von Bedeutung. ${ }^{13}$ Es geht darum, welchen Beitrag Körperlichkeit ${ }^{14}$ und Räumlich-

11 Eine Verbindung zur antiken Konzeption des Topos in der Rhetorik ist offenkundig: Das Topos-Konzept umfasst Vorstellungen und Handlungen, was an einem Ort zu tun sei. Das akzentuiert die Lehre von den Produktionsstadien einer Rede für die Inventio-Phase als Begriff des topischen Denkens, eine aspekt- und themengeleitete systematische Suche nach Argumenten, sowie als produktiven Bestandteil von Mnemotechniken, wonach »das Gedächtnis dadurch gestützt wird, dass man feste Plätze bezeichnet, an denen die Vorstellungen haften [...]. Denn wenn wir nach einer gewissen Zeit an irgendwelche Örtlichkeiten zurückkehren, erkennen wir nicht nur diese selbst wieder, sondern erinnern uns auch daran, was wir dort getan haben«. Quintilian, Ausbildung des Redners, XI, 2.17., hg. von Helmut Rahn, Darmstadt 1995.

12 Deren Basis bildet Konversations- bzw. Gesprächsanalyse. Danach wird soziale Praxis durch Miteinandersprechen hergestellt, die zur Verfügung stehenden routinisierten Gesprächspraktiken werden durch Aufzeige-(display-)Leistungen der Interagierenden sequenziell verstehbar. Ein Gespräch ist in diesem Paradigma durch die Merkmale Konstitutivität, Prozessualität/Sequenzialität, Interaktivität, Methodizität und Pragmatizität gekennzeichnet. Vgl. Arnulf Deppermann, Gespräche analysieren, Wiesbaden 4. Aufl., 2008, 8. Multimodale Interaktionsanalyse erweitert demgegenüber den Gegenstandsbereich: »Wir verstehen Interaktion als multimodale Hervorbringung aller Beteiligten und gehen davon aus, dass alle den Interaktionsbeteiligten zur Gestaltung der Interaktion zur Verfügung stehenden Modalitäten theoretisch zunächst einmal gleichwertig sind.« Vgl. Arnulf Deppermann / Reinhold Schmitt, Koordination. Zur Begründung eines neuen Forschungsgegenstandes, in: Koordination. Analysen zur multimodalen Interaktion, hg. von Reinhold Schmitt, Tübingen 2007, 15-54, 21. Ausführlicher vgl.: Arnulf Deppermann, Multimodal Interaction from a Conversation Analytic Perspective, in: Journal of Pragmatics 46 (2013) 1-7; Reinhold Schmitt, Positionspapier. Multimodale Interaktionsanalyse, in: Ko-Konstruktionen in der Interaktion. Die gemeinsame Arbeit an Äußerungen und anderen sozialen Ereignissen, hg. von Ulrich Dausendschön-Gay / Elisabeth Gülich / Ulrich Krafft, Bielefeld 2015, 43-51.

13 Heiko Hausendorf, Interaktion im Raum. Interaktionstheoretische Bemerkungen zu einem vernachlässigten Aspekt von Anwesenheit, in: Sprache intermedial, hg. von Arnulf Deppermann / Angelika Linke, Berlin 2010, 163-197.

14 Der Anhaltspunkt räumlicher Orientierung ist dabei der Körper selbst und dessen Positionierung. Die Origo (Begriff nach Karl Bühler) als »Navigationszentrum« vereint alle situationsbezogenen Elemente des Sprechens. Andreas Mahler, Topologie, in Jörg Dünne / 
keit zur Interaktionskonstitution leisten, die sich als Aufgabe der Situierung von Interaktion stellt. Diese zeigt sich in der Verschränkung mehrerer Aspekte von Raum, der sich als Wahrnehmungsraum, als Bewegungsraum, als Handlungsraum sowie als Spielraum ausdifferenzieren lässt. ${ }^{15}$ Raum als Wahrnehmungsraum stellt die Interaktionsaufgabe, aus der Fülle des Wahrnehmbaren die für die Interaktion relevanten Wahrnehmungen auszuwählen und zu etablieren (in Hausendorfs Terminologie »Ko-Orientierung« herstellen). Raum als Bewegungsraum stellt die Aufgabe, die Interaktion auch mittels Motorik und Kinesik zu konstellieren, zu eröffnen und $\mathrm{zu}$ beenden, es ist »Ko-Ordinierung« herzustellen. Raum als Handlungsraum fügt die Sprache hinzu, da es notwendig ist, sich auf etwas zu beziehen, was im sozialen Handlungsraum sinnvoll ist, somit wird »Ko-Operation« zur relevanten interaktiven Anforderung. Raum als Spielraum lässt sich als Bühne verstehen, hier werden Benutzbarkeitshinweise ${ }^{16}$ relevant.

Raum als Ressource für die Interaktion anzusehen, heißt auf der analytischen Ebene $^{17}$ ihn gleich zu behandeln wie alle anderen Ressourcen, also Sprache und Körper $^{18}$ auch. Raum ist auch eine Ressource für die Interaktion als sozial instituti-

Andreas Mahler, Berlin 2015, 17-29, insb. 21. Das Ich-hier-jetzt-System als deiktisches Zentrum der Sprechsituation kennzeichnet jede Interaktion als durch ein Ich-(Du), die Anwesenheit von Personen, durch den Raum als ein Hier sowie durch die Zeit als ein Jetzt bestimmt. Vgl. Anja Stukenbrock, Deixis in der face-to-face-Interaktion, Berlin 2015,12 .

15 Hausendorf, Interaktion im Raum (wie Anm. 13) 172, 175-190.

16 Vgl. zum Konzept der Benutzbarkeitshinweise ausführlicher im Abschnitt Fallanalysen.

17 Dass das Miteinandersprechen immer von Raum umgeben ist, ist in gesprächsanalytischen Arbeiten wegen seiner Unumgänglichkeit oft vorausgesetzt worden. Die technische Entwicklung der Aufzeichnungsmittel von Gesprächen bzw. Interaktion brachte hierzu die Veränderung: Erlaubten traditionelle Audioaufzeichnungen - lange Zeit das alleinige Erhebungsformat - raumbezogene Aspekte allenfalls als Kontextualisierungshinweise zu fassen, wird mit der Videoaufzeichnung als Standard bei der Korpuserhebung in interaktionsanalytischen Studien Visualität wichtig. Dabei entspricht in der Gesprächsanalyse der Zeit - anders als dem Raum - eine eigene Analysekategorie, die Sequenzialität, womit die zeitlich-prozessuale Organisation von Gesprächspraktiken im Sinne einer konstitutionslogisch relevanten Abfolge von Aktivitäten gemeint ist. Vgl. zur Analysekategorie der Sequenzialität Deppermann, Gespräche (wie Anm. 12). Videodaten hingegen zeigen, dass Sequenzialität nur ein Mechanismus zur Herstellung von komplexer, multimodaler Interaktion ist, sie muss um den Mechanismus der Simultaneität ergänzt und mit ihm verbunden werden. Vgl. Deppermann / Schmitt, Koordination (wie Anm. 4) 30.

18 Der analytische Blick auf den (kommunizierenden) Körper ist selbst immer kulturell und historisch eingebettet und unterliegt so der Veränderung, worauf Linke in ihrer Untersuchung von Konversationsbüchern und Schriften zur praktischen Rhetorik hingewiesen 
onalisierter Raum, wie er sich aus der funktionalen Differenzierung der Gesellschaft ableitet. Dessen Voraussetzung ist die Wirksamkeit räumlicher Arrangements, sie weisen Affordanzen ${ }^{19}$ bzw. Valenzen auf. So gibt es Räume, »die Verhalten und Handlungen sowie Kommunikationen prägen und vorstrukturieren: etwa in der Kirche, auf Behörden, in Seminarräumen oder Wartezimmern. [...] Die Vorlesung des Professors entfaltet keineswegs überall ihre Wirkung, sondern muss durch räumliche wie zeitliche Arrangements entsprechend vorbereitet und flankiert werden. $\ll^{20}$ Hausendorf und Schmitt sprechen in diesem Zusammenhang von Interaktionsarchitektur:

»Interaktionsarchitektur steht für die Frage, wie die Architektur von Räumen Interaktion (wenn auch nicht determinieren und verhindern, so doch) ermöglichen und nahelegen kann und wie man diese interaktionsarchitektonischen Implikationen empirisch rekonstruieren kann. Unter >Architektur verstehen wir dabei heuristisch all das, was vom gebauten Raum (aus Stein, Beton, Holz, ...) über den gestalteten Raum (`Innenarchitektur $)$ bis zum ausgestatteten Raum (z.B. Technik, Dekoration) reicht. $\ll^{21}$

Für die Interaktion kommt dem Raum die Funktion einer Entscheidungshilfe in Bezug auf die Art der Situation, die erwartbaren Handlungen und Interaktionsabläufe zu, da bestimmten Räumen im gebauten Raum bestimmte Tätigkeiten zuordenbar sind wie beispielsweise schlafen im Schlafzimmer oder lernen im Seminarraum, was nicht heißen muss, dass sie auch nur dort durchgeführt werden. ${ }^{22}$ Durch diese Handlungen wird Räumen Bedeutung zugeschrieben, die nicht jedes Mal neu vorgenommen wird, es gilt vielmehr, dass »vorgegebene räumliche Arrangements ge-

hat. Vgl. Angelika Linke, Historische Semantik des Leibes in der Kommunikation. Zur Dynamisierung von Körper und Sprache im ausgehenden 17. und 18. Jahrhundert, in: Sprache intermedial, hg. von Arnulf Deppermann / Angelika Linke, Berlin 2010, 129162; ähnlich: Lily Tonger-Erk, Actio. Körper und Geschlecht in der Rhetoriklehre, Berlin 2012.

19 Das ursprünglich aus der Biologie stammende, von Bildwissenschaft und UsabilityForschung adaptierte Konzept der Affordanz pointiert den Angebotscharakter von Erscheinungsweisen der situativen Umgebung für kommunikative Handlungsmöglichkeiten. Vgl. Hausendorf / Schmitt, Interaktionsarchitektur (wie Anm. 6) verknüpfen das mit dem Konzept der Benutzbarkeitshinweise.

20 Schroer, Räume (wie Anm. 3) 176.

21 Hausendorf / Schmitt, Interaktionsarchitektur (wie Anm. 6) 3.

22 Schroer, Räume (wie Anm. 3) 176, 288. Gerade die effektive Umnutzung eines Seminarraums zu einem Filmvorführraum akzentuiert, welche Aspekte von Räumlichkeit verändert werden müssen, um die neue Nutzung zu gestalten. Vgl. Hausendorf, Tische und Bänke (wie Anm. 7). 
rade von Situationsdefinitionen [entlasten], weil die Bedeutungen und Wertigkeiten der Akteure bereits in sie eingeschrieben sind. $\ll^{23}$ Diese Entscheidungshilfe kann der Raum für die in der Institution handelnden Körper aber nur dann sein, wenn soziale und interaktive Routinen geprägt worden sind, d.h. körperliche Fertigkeiten durch Habitualisierung zu Gewohnheitswissen geworden sind, dem Routinisierung zugrunde liegt. ${ }^{24}$ Im Seminarraum findet sich also ein spezielles räumliches Arrangement, das ein bestimmtes Verhalten nahe legt, ein anderes unterdrückt und zugleich funktional prägt bzw. geprägt wird, zugleich haben die darin handelnden Menschen zumeist eine Institutionenbiografie. Das ist Wissen, auch habitualisiertes Körper- und Raumwissen, das zur Interaktionskonstitution zur Verfügung steht.

\section{HANDELN IM GEBAUTEN RAUM DER UNIVERSITÄT: DAS SEMINAR}

Die Bildungs- und Lehrfunktion der Universität realisiert sich in Lehrveranstaltungen. Dieser Funktion entsprechend dienen sie durch Erkenntnisdiskussion, Wissenspräsentation und -evaluation der Wissensvermittlung. Gespräche als eine Form der institutionellen, öffentlichen und weitgehend festgelegten Kommunikation in der Universität haben als Lehrgespräch bzw. als maieutischer Dialog eine lange Geschichte, die in Platons Akademie ihren Anfang nimmt. In diesen Gesprächen lassen sich Handlungen und Rahmenbedingungen, die direkt der Wissensvermittlung dienen, von solchen Handlungen und Rahmenbedingungen unterscheiden, die die Bedingungen für gelungene Wissensvermittlung herstellen. ${ }^{25}$ Die dominanten Formate universitärer Lehre sind die Vorlesung und das Seminar. Letzteres ist die akademische Lehrform, die sich durch Gesprächshaftigkeit sowie studentische Partizipation auszeichnet.

Das Seminar wird zumeist doppelt bestimmt: »Ein Seminar ist ein Ort zur Ausbildung junger Menschen, die entsprechende Institution sowie die in ihr praktizierte, auf Nachhaltigkeit angelegte Form der Lehre. ${ }^{26}$ Danach geht es um einen Ort, der zu einer Aktivität einlädt bzw. verpflichtet, gleichzeitig wird durch diese Hand-

23 Schroer, Räume (wie Anm. 3) 177.

24 Hubert Knoblauch, Kulturkörper, in: Soziologie des Körpers, hg. von Markus Schroer, Frankfurt am Main, 2. Aufl., 2012, 92-113, 102.

25 Wolfgang Sucharowski, Gespräche in Schule, Hochschule und Ausbildung, in: Text- und Gesprächslinguistik, hg. von Klaus Brinker / Gerd Antos / Wolfgang Heinemann / Svend F. Sager, Berlin 2001, 1566-1576.

26 Ulrich Breuer / Matthias Emrich, Seminar, in: Über die Praxis des kulturwissenschaftlichen Arbeitens, hg. von Ute Frietsch / Jörg Rogge, Bielefeld 2013, 376-381, 376. 
lungen der Ort konstituiert und erkennbar. Die Bezeichnung Seminar geht auf lat. semino: säen und das Seminarium als Pflanzschule zurück:

»seminar, n., nach WEIGAND ${ }^{4}$ 2, 695 im 17. jahrh. aus lat. seminarium, pflanzstätte, entlehnt, anstalt für geistige ausbildung, so von lehrern, schullehrerseminar, dazu seminarist, besucher, zögling eines solchen schullehrerseminars, seminardirector, - lehrer, die an solchem seminar angestellt sind. seminare an universitäten, einrichtungen zu methodischer wissenschaftlicher ausbildung von studenten in persönlichem verkehr mit professoren: classisch - philologisches, deutsches, englisches, romanisches, historisches seminar. $\ll^{27}$

Betont wird bereits im Grimm'schen Wörterbuch, wie auch später immer wieder, die anwesenheitsbasierte Gemeinschaft von Studierenden und Lehrenden. Diese Gemeinschaft zeichnet eine besondere Verbindung aus und die auf Nachhaltigkeit angelegten Bemühungen sind Ausdruck derselben. Seminare sind in diesem Sinn »Veranstaltungen, die als Pflanzstätten, als Stätten der Aussaat zu verstehen sind, wo der Samen des lebendigen Geistes ausgebracht wird, und zwar in kollektiven Lernprozessen an einem Ort, an dem in solidarischer Arbeit Erkenntnis voranschreitet. $\ll^{28}$ Das akademische Seminar ist der Raum des innovativen Tätigkeitstyps und des forschenden Lernens und kann als "problem- und forschungsorientierter, institutionell abgestützter Modus intensivierender Wissenskommunikation $\ll^{29}$ verstanden werden. Es kommt in dieser Art fast ausschließlich in den Geisteswissenschaften vor, wobei das philologische Seminar - eine deutsche Erfindung aus dem 19. Jahrhundert - zugleich lange als Modellfall der Verbindung von Forschung und Lehre galt. ${ }^{30}$

Der thematische Zuschnitt eines Seminars ist problemorientiert und vertiefend, das erlaubt, das Seminar den Fachdisziplinen zuzuordnen. Ausdruck dieser Zuordnung ist ferner, dass im Seminar die Fähigkeiten im Umgang mit fachspezifischem

27 Deutsches Wörterbuch von Jacob und Wilhelm Grimm, 16 Bde. in 32 Teilbänden, Leipzig 1854-1961. Quellenverzeichnis, Leipzig 1971, Online-Version vom 5.6.2016.

28 Ulrich Wyss, Zur Tradition der Vorlesung, in: Dusini / Miklautsch, Vorlesung (wie Anm. 1) 177-192, 186. Solche hyperbolisch anmutenden Gärtnerei- bzw. Naturmetaphern für das Lernen sind tradiert, wie überhaupt Raumsemantiken die Bilder von Erziehung und Lernen prägen: der Erzieher als Gärtner, das Führen und Wachsenlassen oder auch das Unkrautbeseitigen; vgl. Martin Nugel, Erziehungswissenschaftliche Diskurse über Räume der Pädagogik, Wiesbaden 2014, 103 ff.

29 Breuer / Emrich, Seminar (wie Anm. 26) 376.

30 Gert Schubring, Kabinett - Seminar - Institut. Raum und Rahmen des forschenden Lernens, in: Berichte zur Wissenschaftsgeschichte 23 (2000) 269-285, vgl. 269; Thomas Finkenstaedt, Der Universitätslehrer, in: Geschichte der Universität in Europa, hg. von Walter Rüegg, Bd. 4, München 2010, 153-189, insb. 177. 
Wissen sowie mit fachspezifischen Methoden geschult werden. Auf diese Weise werden Studierende in einen forschenden Habitus eingeübt: Die viel beschworene Einheit von Forschung und Lehre realisiert sich stark im Seminar. Wenngleich das Seminar aus diesem Grund zwar als wichtiger als die Vorlesung angesehen wird, sind beide Veranstaltungstypen jedoch mit unterschiedlich hoher akademischer Wertschätzung verknüpft. ${ }^{31}$

Aus der folgenden Definition lassen sich die für die Interaktionsanalyse wesentlichen Merkmale eines Seminars ableiten:

»Das Seminar führt eine limitierte Anzahl von Studierenden mit einer examinierten Lehrkraft über einen begrenzten Zeitraum in einem mit Medien (Tafel, Projektor, Beamer etc.) ausgestatteten Unterrichtsraum zusammen, wo sich die Teilnehmer während der gemeinsamen $\rightarrow$ Sitzungen in frei gewählter Form über ein vorab angekündigtes fachliches Thema austauschen. $\ll^{32}$

Diese Merkmale sind denen der Vorlesung ${ }^{33}$ entgegengesetzt. Das Merkmal des Zugangs bedeutet für das (zumeist semestral andauernde) Seminar nicht Offenheit, sondern quantitative Beschränktheit in der Anzahl der Studierenden. Der Zugang ist auch qualitativ beschränkt, indem er sich auf Mitglieder der Universität bezieht, die zudem in der Lage (nachgewiesen durch verschiedene Voraussetzungen und den Besuch vorgängiger Lehrveranstaltungen) sein müssen, thematisch-fachlich folgen zu können. Über den Zugang als Aufnahme oder Ablehnung entscheidet die Lehrperson. Zugehörigkeit zum Seminar wird indes durch leiblich-persönliche Anwesenheit hergestellt, wobei Abwesenheit erlaubt ist.

Die Interagierenden im Seminar sind Studierende und Lehrpersonen in Funktionsrollen der Institution Universität. Für die Studierenden gilt, dass es sich hier um den dritten Bildungsschritt ihrer Bildungsbiografie handelt, sie sind als bildungs-, wenn auch zunächst nicht als universitätserfahren zu kennzeichnen. Mit diesen

31 So gilt die Vorlesung als ungleich glorioser, was auch mit dem institutionellen Zugang, eine Vorlesung halten zu dürfen, zu tun hat (hingewiesen sei auf Lehrstuhl und venia legendi). Vgl. Werner Michler, Die Vorlesung als soziales Ereignis, in: Dusini / Miklautsch, Vorlesung (wie Anm. 1) 23-40, insb. 24.

32 Breuer/Emrich, Seminar (wie Anm. 26) 376.

33 Die Vorlesung erfolgt zumeist vor einer großen Gruppe, der dafür tradierte Ort ist der Hörsaal, dessen Einrichtung klare, eindeutige Benutzbarkeitshinweise macht. Das Rederecht ist institutionell geregelt, d.h. die Lehrperson hat es durchgehend. Der thematische Zuschnitt ist eher breit und überblicksmäßig. Dabei wird die Vorlesung verschieden konzeptualisiert, beispielsweise als »säkularisierte Predigt«, Wyss, Tradition der Vorlesung (wie Anm. 28) 188. Sie kann auch als performative Gattung mit Unterhaltungspotenzial angesehen werden. Vgl. Sybille Peters, Der Vortrag als Performance, Bielefeld 2011. 
Funktionsrollen sind außerdem verschiedene Arten der Interaktionsbeteiligung verbunden. Die Lehrperson hat, neben der moderativen Gesprächsleitung, auch die permanente Zielorientierung - die Erreichung von Bildungszielen - der gemeinsamen Arbeit im Seminar abzusichern. Idealtypisch gilt im Seminar als einer akademischen Praxis jedoch die Gleichstellung der am Seminar Beteiligten, d.h. »Autorität kann im wissenschaftlichen Seminar allein die schärfere Beobachtung, die präzisere Beschreibung und das überzeugendere Argument beanspruchen. $\ll^{34}$

Der Raum in der Universität, in der ein Seminar stattfindet, zeichnet sich durch fixierte und mobile Erscheinungsformen des Mobiliars aus, erwartbar sind typische Objekte wie Tafel, Computer, Beamer und Tische/Stühle. Die Einrichtung des Raums hat den Zweck, die Interagierenden in einen den Bildungszielen förderlichen Kontakt zu versetzen. Der gebaute Raum ist hergerichtet für diesen Zweck und verändert sich durch die Interaktion zunächst nicht. Die Bewegungsmöglichkeiten und die Bewegungsdynamik im Seminar sind für die Studierenden zumeist eingeschränkt, d.h. sie (müssen) sitzen. Die Lehrperson hingegen kann zwischen Sitzen, Stehen und Gehen wählen.

Nach der Darstellung des theoretischen Rahmens sowie einem Blick auf das Seminar in der Perspektive der Hochschulforschung soll im Folgenden das empirische Material vorgestell und der Interaktionstyp Auswertungsgespräch, der Teil der seminarbezogenen Interaktionstypen ist, näher erläutert werden.

\section{DATENBASIS}

Die empirische Basis der Fallanalysen bildet das Korpus Auswertungsgespräche (im Folgenden: K_Awg), ein von der Autorin über den Zeitraum eines akademischen Jahres systematisch erhobenes Korpus authentischer Lehr-/Lerninteraktion in universitären Seminaren einer Germanistik. ${ }^{35}$ Es umfasst drei Datenarten: erstens ca. 120 Stunden videographierte ${ }^{36}$ Auswertungsgespräche aus germanistischen

34 Breuer / Emrich, Seminar (wie Anm. 26) 376-377.

35 Schwarze, Auswertungsgespräch (wie Anm. 8); Cordula Schwarze, Angemessenheitsverhandlungen in Auswertungsgesprächen in der Hochschullehre, in: Aptum 2 (2015) 190199.

36 Genutzt wird die in der soziologisch informierten Interaktionsanalyse entwickelte Form der Videographie, die $»$ Verbindung von Videoanalyse und Ethnographie, also die interpretative Analyse von Videodaten kommunikativer Handlungen, die im Rahmen eines ethnographischen Erhebungsprozesses aufgezeichnet werden.« René Tuma / Bernt Schnettler / Hubert Knoblauch, Videographie, Wiesbaden 2013, 10. Interpretativ ist dabei als explizierend und rekonstruierend $\mathrm{zu}$ verstehen, insofern ist die Anschlussfähigkeit zum Vorgehen der multimodalen Interaktionsanalyse gegeben. 
Lehrveranstaltungen, zweitens das gesamte ethnografische Zusatzmaterial zu diesen Lehrveranstaltungen sowie Feldnotizen und drittens eine mehrstündige Fokusgruppendiskussion mit sieben Lehrpersonen aus den videographierten Auswertungsgesprächen. Die videographierten Lehrveranstaltungen haben Themen der Rhetorik sowie Textproduktion zum Gegenstand und den Erwerb professionsbezogener kommunikativer Kompetenz als Qualifikationsziel. Als Interaktionstyp ist das Auswertungsgespräch durch sprachreflexives und sprachanalytisches Handeln gekennzeichnet, dabei stehen die reflexive Bearbeitung von Konvergenzen und Divergenzen auf der Ebene der wahrnehmungs- und wissensgestützten Beobachtung, Analyse und Beurteilung sowie das Aufzeigen von Handlungsalternativen anhand eines Schreibproduktes oder mündlichen rhetorischen Ereignisses eines anwesenden Lernenden durch die Seminargruppe im Mittelpunkt. Der konstituierende Faktor eines Auswertungsgesprächs ist das Vorliegen eines Auswertungsobjekts, auf das sich die auswertenden Handlungen beziehen lassen. Das Auswertungsobjekt der nachfolgenden Fallanalysen wurde in einem Seminar zur wissenschaftlichen Rhetorik generiert, es ist dies eine Rede, ${ }^{37}$ in der eine germanistisch-linguistische Theorie medienunterstützt mit dem Ziel der umfassenden Information des Publikums vorgestellt wurde.

Da Raumanalysen auf Videodaten beruhen, kommt dem Kamerahandeln in den Daten eine wesentliche Rolle zu. Die Kamera in dieser Erhebungssituation ist zwar eine fixierte Kamera, wird aber mobil und subjektiv eingesetzt. Sie fokussiert Handlungszentren und agiert zunächst sprecher_innenzentriert, indem sie sich auf den/die primäre_n Sprecher_in richtet, zugleich rücken aber auch diejenigen Beteiligten in den Blick, die sich in diesem Moment nicht an der Interaktion beteiligen. An diesem Vorgehen - obwohl in der Erhebungssituation wegen der Invasivität der aufzeichnenden Kamera sowie Gruppen- und Raumgröße unumgänglich - zeigt sich ein Problem wissenschaftlicher Raumbeschreibung: »Als Beobachter und Involvierter ist der Mensch immer schon Teil des von ihm beschriebenen Raums, immer schon im Raum verortet wie vom Raum abhängig, und er erscheint unweigerlich als dessen Element. $\ll^{38}$ Die Daten aus dieser Erhebungssituation sind also

37 Eine solche Rede kann rhetorisch-gattungsbezogen dem genus didascalicum (Gattung der Lehrrede) zugeordnet werden. Darin stehen das Einsichtigwerden in einen Sachverhalt, also Verstehen und Erkenntnis, im Mittelpunkt der rhetorischen Bemühungen, nicht jedoch Handlungsaufrufe oder die Bewertung eines Sachverhalts. Das genus didascalicum wurde von Philipp Melanchthon in das rhetorische System eingefügt. Vgl. Martin Leiner, Genus didascalicum, in: Historisches Wörterbuch der Rhetorik Bd. 10: Nachträge A-Z, hg. von Gert Ueding, Berlin 2012, 329-333, insb. 329. Die Gattung der Lehrrede kann heute, nachdem sie einige Jahrhunderte wenig Aufmerksamkeit erhielt, produktiv an rhetorische Formate im akademischen Feld angeschlossen werden.

38 Mahler, Topologie (wie Anm. 14) 17. 
einperspektivisch, die Standort- und Perspektivengebundenheit von Videoaufnahmen ermöglicht nicht, den Raum im Ganzen zu erfassen, sondern lediglich selektierte Ausschnitte.

Das methodische Vorgehen in den Fallanalysen des vorliegenden Aufsatzes besteht neben der Transkription der Daten ${ }^{39}$ und der Sequenzanalyse auch in einer Standbildanalyse. Das ist ein in der multimodalen Interaktionsanalyse vielfach genutztes Verfahren. ${ }^{40}$ Standbilder werden aus den Videodaten des Korpus extrahiert, ihre Auswahl beruht auf Selektionsentscheidungen der Analysandin. Standbilder frieren Interaktion ein, aber »erzählen aus dieser Perspektive Interaktionsgeschichten: Sie sind retrospektiv (Was ist passiert? Was war hier los?) wie prospektiv (Wie wird es weitergehen? Was kommt als Nächstes?) lesbar. « ${ }^{41}$ Insbesondere für die Analyse von räumlichen und mobiliaren Benutzbarkeitshinweisen scheint das Verfahren ergiebig, denn im Standbild wird die Gestaltung des Raums besser sichtbar und Benutzbarkeitshinweise offerieren deutlicher ihr Potenzial und somit mehrere Lesarten, Interaktionsfortsetzung nahe zu legen. Im nächsten Schritt werden die

39 Die Daten sind sowohl in den Transkripten als auch auf den Bildern anonymisiert worden. Die Transkripte sind an GAT 2 orientiert, zu den Transkriptionskonventionen vgl. Margret Selting et al., Gesprächsanalytisches Transkriptionssystem 2 (GAT 2), in: Gesprächsforschung - Online-Zeitschrift zur verbalen Interaktion 10 (2009) 353-402.

40 Diese Art der Standbildanalyse wurde etabliert von Reinhold Schmitt (z.B. Körperlichräumliche Aspekte, wie Anm. 5) und Heiko Hausendorf (z.B. Tische und Bänke, wie Anm. 7). Ähnlich auch: Stukenbrock, Deixis (wie Anm. 14) 25 ff. Dazu muss jedoch darauf verwiesen werden, dass das Problem der Transkription, Vertextung und angemessenen Repräsentation von visuellen Daten in der Analyse derzeit breit und kontrovers diskutiert wird, wobei vor allem das Verhältnis von Transkript und Bild/Video als noch nicht hinreichend methodologisch durchdrungen gilt. Konkret werden auch gute Gründe vorgebracht, um das Verfahren der Nutzung des stehenden Bildes bei der Analyse laufender Bilder (Videos) wegen ihres kategorialen Unterschieds als unzureichend abzulehnen. So z.B. Jo Reichertz, Das vertextete Bild. Überlegungen zur Gültigkeit von Videoanalysen. in: Transkription von Video- und Filmdaten in der Qualitativen Sozialforschung, hg. von Christine Moritz, Wiesbaden 2014, 55-72; oder auch: Christine Moritz, Vor, hinter, für und mit der Kamera: Viergliedriger Video-Analyserahmen in der Qualitativen Sozialforschung, in: Transkription von Video- und Filmdaten in der Qualitativen Sozialforschung, hg. von Christine Moritz, Wiesbaden 2014, 17-54; Christian Heath / Jon Hindmarsh / Paul Luff, Video in Qualitative Research. Analyzing Social Interaction in Everyday Life, Los Angeles 2010; Ulrike Bohle, Approaching Notation, Coding, and Analysis from a Conversation Analysis Point of View, in: Body - Language - Communication. An International Handbook on Multimodality in Human Interaction, Bd. 1, hg. von Cornelia Müller et al., Berlin 2013, 992-1007.

41 Hausendorf, Tische und Bänke (wie Anm. 7) 145. 
Standbilder mit den Transkripten bzw. den Sequenzanalysen verknüpft, da die dokumentierte Interaktion der gemeinsame Bezugspunkt ist.

\section{FALLANALYSEN}

Im nachfolgenden Abschnitt werden exemplarisch drei Fallanalysen präsentiert. Dabei wird das Augenmerk auf alle drei Interaktionsbeteiligten des Seminars Raum, Lehrperson und Studierende - gerichtet. Die erste Frage an die Daten ist die nach der Art des Zusammenhangs der Lösung interaktiver Aufgaben durch relevant gesetzte Aspekte von Raum. Zu spezifizieren sind die Fragen folgendermaßen:

- Was wird durch räumliche Benutzung und Begrenzung ermöglicht oder verhindert?

- Wie werden Benutzbarkeitshinweise im Sinne einer räumlichen Ressource für die Situierung als ein Seminar mit spezifischen pädagogischen Zielen ausgenutzt? Welchen Benutzbarkeitshinweisen folgen Studierende und Lehrperson? Wie verschränkt sich das mit der Interaktion?

- Welcher Zusammenhang besteht zwischen körperlichen und räumlichen Konstellationen und wie ist er beschreibbar?

In den Analysen zeigt sich die Raumnutzung zunächst unauffällig und vertrautheitskonform, mobiliare Benutzbarkeitshinweise werden die Interaktion fördernd und unterstützend verwendet, es greifen verschiedene Ressourcen ineinander. Dennoch sind sie interessant und aussagekräftig, weil solche Fälle etwas über die produktive Alltäglichkeit solcher Situierungen der Interaktion offenbaren. ${ }^{42}$

Im ersten Beispiel wird als Grundlage aller weiteren Standbild- und Interaktionsanalysen die Interaktionsarchitektur ${ }^{43}$ des Seminarraums nachgezeichnet. Es soll gezeigt werden, welche Benutzbarkeitshinweise der (Seminar-)Raum und seine Möblierung zunächst unabhängig von der Interaktion machen. Benutzbarkeitshinweise stellen »Anknüpfungspunkte für Wahrnehmung, Bewegung und Handlung zur Verfügung. Im Alltag werden sie häufig wie selbstverständlich verstanden und körperlich `beantwortet««. ${ }^{44}$ Während das erste Beispiel zeigt, was durch Architek-

42 Zweifellos ist eine solche Aussage kulturrelativ: Beispielsweise zeigen Videodaten aus Lehr-/Lernsituationen an einer Universität in Laos (Korpus des Projekts Mündlichkeit und Interaktionskompetenz im DaF-Unterricht, Leitung: Cordula Schwarze und Ines Bose), dass permanentes Sitzen seitens der Studierenden während der Lehrveranstaltung keine zwingende und ausschließliche Handlungsweise sein muss.

43 Hausendorf / Schmitt, Interaktionsarchitektur (wie Anm. 6) 3.

44 Hausendorf, Tische und Bänke (wie Anm. 7) 139. 
tur erwartbar gemacht wird, rekonstruieren die Beispiele zwei und drei die stattgehabte Interaktion. Die Basiskonzepte der Interaktionsarchitekturanalyse wie Sichtbarkeit, Hörbarkeit, Begehbarkeit, Begreifbarkeit und Verweilbarkeit, ${ }^{45}$ die zunächst unabhängig von der Interaktion sind, strukturieren erkenntnisleitend die Auswahl des ersten Beispiels vor. Die Implikationen des Raums und seiner Benutzbarkeitshinweise kommen nur vertrautheits- und wissensabhängig zur Geltung ${ }^{46}$ und müssen mit sozialer Praxis verknüpft werden, was in den nachfolgenden Beispielen gezeigt werden soll. Im zweiten Beispiel soll der Fokus auf die Lehrperson und deren Handeln gelegt werden. Es kann gezeigt werden, wie die Lehrperson aus dem Angebot an technischen Objekten gestisch auswählt und damit der interaktiven Aufgabe der Koorientierung der Interagierenden sowie der Kohärenzherstellung für das Seminar versucht nachzukommen. Im dritten Beispiel wird gezeigt, wie aus der Gruppe der Studierenden heraus eine Binnendifferenzierung des Raums als Interaktionsraum hergestellt wird, indem eine Interaktionsdyade eröffnet wird. Diese zeichnet sich dadurch aus, dass sie zwar einerseits klar als Dyade sichtbar wird, es andererseits aber den Interagierenden gelingt, die Dyade als Teil der Lehr/Lerninteraktion im Seminar und speziell als Auswertungsaktivität zu markieren.

\section{Fallanalysen Beispiel 1: Der Raum}

Der gebaute Raum ist in diesem Fall auch ausgestatteter Raum und liefert damit die Kandidaten für eine räumliche, im nächsten Schritt auch räumlich-körperliche Konstellationsanalyse. Die "genuine Lesbarkeit des Raumes $«{ }^{47}$ ist die Voraussetzung für die Interaktionsaufgabe der Situierung. Es reicht aber nicht aus, auf die materielle Ausformung des Raums nur zu verweisen. Diese muss vielmehr präzise analysiert werden: »Es geht nicht nur darum zu sehen, wie der Raum sozial hergestellt wird, sondern auch darum zu berücksichtigen, was der Raum selbst vorgibt. Das hat nun nichts mit Raumdeterminismus zu tun, sondern damit, dass räumliche Arrangements nicht ohne Wirkung auf unser Verhalten bleiben. Die Fülle möglicher Verhaltensweisen wird durch Raum selektiert und damit Kontingenz bewältigt. $\ll^{48}$

Die Abbildung 1 zeigt den Grundriss des Raums, in dem das videographierte Seminar stattfand, sie zeigt auch die Möblierung zum Zeitpunkt der Erhebung.

45 Hausendorf / Schmitt, Interaktionsarchitektur (wie Anm. 6) 12 und 17.

46 Hausendorf / Schmitt, Interaktionsarchitektur (wie Anm. 6) 13.

47 Hausendorf, Interaktion im Raum (wie Anm. 13) 172.

48 Schoer, Räume (wie Anm. 3) 178; ähnlich auch Hausendorf / Schmitt / Mondada, Raum (wie Anm. 3). 
Abbildung 1: Grundrissskizze des Raums in der Erhebungssituation.

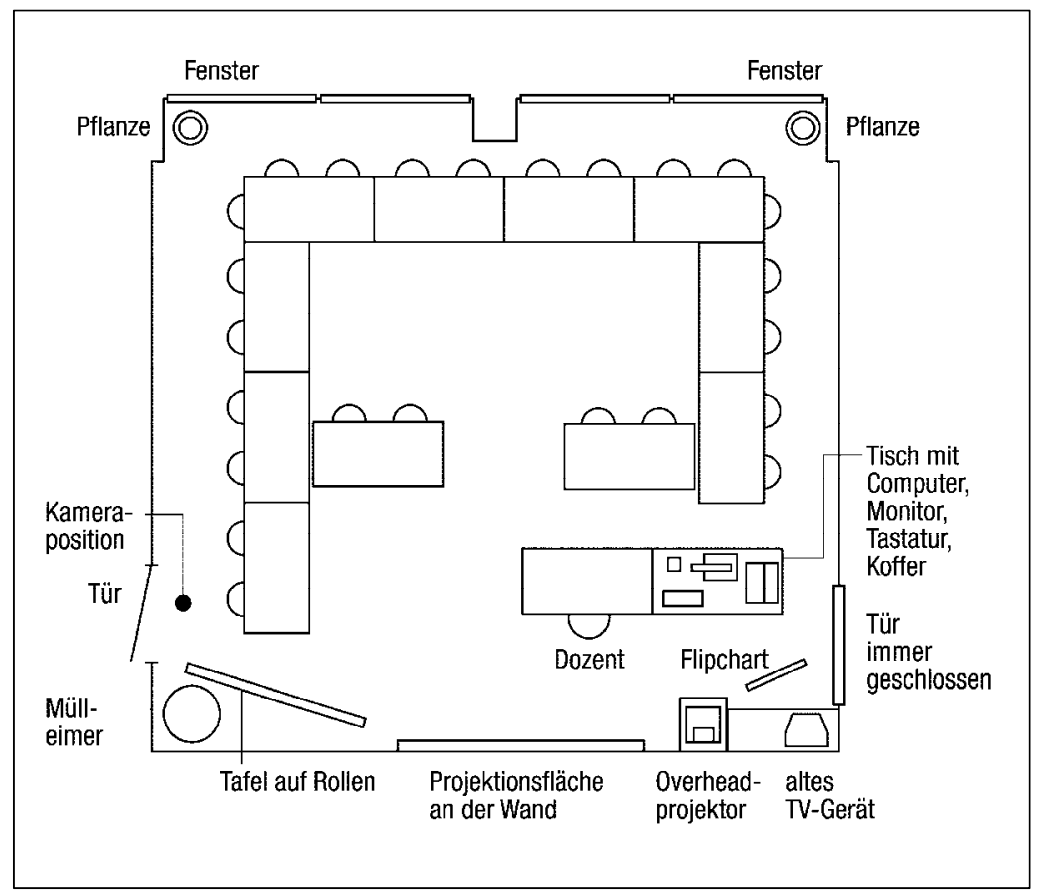

Quelle: Graphik von der Autorin (K_Awg: R_EP_2_1).

Türen sind unter dem Aspekt der Begehbarkeit wichtig, sie stellen eine »Betretbarkeits- und Verlassbarkeitsschwelle« dar. ${ }^{49}$ Der Raum hat zwei gegenüberliegende Türen. Beide Türen sind während des Seminars geschlossen. Als Ein- und Ausgang wird nur eine Tür genutzt, das ist die, vor der die Kamera steht, die andere Tür ist unbenutzt und bekommt daher den Charakter eines Hintereingangs. Die zum Betreten genutzte Tür ist von außen beschriftet. Daran ist der wochenaktuelle Plan mit der Raumbelegung befestigt, der Plan enthält den Lehrveranstaltungsnamen und -nummer, den Namen der Lehrveranstaltungsleitung sowie die exakten Zeiten der Lehrveranstaltung. Von außen und bei geschlossener Tür ist also feststellbar, was im Raum gerade passiert, d.h. wie es institutionell bezeichnet wird und wer die handelnde Lehrperson ist. Dieser Plan, an die Zeit gekoppelt, dient der Regelung der Betretbarkeit und bekräftigt so die geschlossene Tür in dem Sinne, dass während der Lehrveranstaltung das Betreten nicht (einfach so) erlaubt ist. ${ }^{50}$

49 Hausendorf, Tische und Bänke (wie Anm. 7) 158.

50 Der Raumplan als Beschriftung des Seminarraums weist Ähnlichkeiten mit der Beschriftung von Kunstwerken im Museum auf, deren Merkmale von Heiko Hausendorf, Kunst- 
Im Raum befinden sich mehrere Tische, bis auf einen sind es mobile, hellgraue, rechteckige Tische mit vier Beinen an den Ecken. Ein Großteil der Tische ist in UForm gestellt, zwei Tische sind an den Innenseiten des U nach innen gestellt und zwei weitere Tische stehen mit einem kleinen Abstand davor. Der Abstand markiert eine Trennung und macht damit Territorien sichtbar, die mit unterschiedlichen Begehbarkeitsmöglichkeiten ausgestattet sind. Die U-Form richtet auf ein Vorn im Raum aus, auf ein zentrales Element, was dort sichtbar sein soll. Das ist zum einen die Lehrperson und deren Territorium (Tisch, technische Geräte), zum anderen die für alle sichtbare Projektionsfläche sowie die Tafel. Der Benutzbarkeitshinweis der flexiblen Aufstellbarkeit der Tische wird hier genutzt, um eine zweckdienliche Tischaufstellung zu schaffen. Die Stellung der Tische kann demzufolge als eine Antwort auf Anforderung des Seminars angesehen werden, sie ermöglicht gemeinsame Aufmerksamkeitsausrichtung und damit Koorientierung (und ggf. Koordination) auf die Lehrperson ${ }^{51}$ sowie auf Projektionsfläche, Redeort und Tafel/Whiteboard. Das Potenzial in Bezug auf die Benutzung der mobilen Tische besteht darin, dass sie durch ihre Höhe die Handlung des Sitzens nahe legen und durch ihr Vorhandensein das Herumlaufen eher ausschließen. Zugleich offerieren sie Flächen zum Ablegen und Schreiben; in Bezug auf die Sitzplatzwahl ermöglichen sie die Zuordnung zu präferierten Gruppen. In dem als Vorn bezeichenbaren Territorium stehen zwei Tische nebeneinander, die wissensabhängig als Tische der Lehrperson bezeichnet werden können. Sie haben durch ihre Platzierung die höchste Sichtbarkeit von allen. Einer der beiden Tische ist der einzige am Boden fixierte Tisch und

kommunikation, in: Textsorten, Handlungsmuster, Oberflächen, hg. von Stephan Habscheid, Berlin 2011, 499-535, herausgearbeitet wurden. Beim Raumplan an der Tür zum Seminarraum ist vertrautheitsabhängig offensichtlich, dass es eine Schild-TürRelation gibt, welche die kommunikative Handlung des Bezugnehmens, also eine Antwort auf die Frage Worum geht es?, ermöglicht. Das Schild an der Tür ist jedoch nur lesbar und erfüllt, wie die Beschriftungen von Kunstwerken auch, seine Funktion nur genau an dieser Tür, also bei geteilter Wahrnehmung. Damit nimmt das Türschild eine den Kunstwerksbeschriftungen ähnliche Zwischenstellung von Schriftlichkeit ein, denn es dient gerade nicht in erster Linie der Verdauerung.

51 Diese Anordnung der Tische sowie der sich auch daraus ergebenden territorialen Aufteilung im Raum macht das Konzept der Fokusperson und deren Anwendung auf die Lehrperson nachvollziehbar. Eine Fokusperson ist »eine[n] Interaktionsbeteiligte[n], die/der aufgrund von Status, Funktion oder Rolle in bestimmten Kontexten kontinuierlicher Bezugspunkt von Monitoring-Aktivitäten anderer Beteiligter ist.« Deppermann / Schmitt, Koordination (wie Anm. 4) insb. 35. Der Lehrperson steht in der konkreten Unterrichtssituation die Gesamtheit der Studierenden gegenüber (teils räumlich unterstützt), daher ist sie als fokussierte Person stets im Wahrnehmungsfeld der Studierenden und zugleich Mittelpunkt ihrer Wahrnehmung. 
der einzige mit fest montierten Geräten (Computer mit Bildschirm und Tastatur, Bedieneinheit des Beamers, zwei Lautsprecherboxen) darauf. So wird er als Arbeitstisch sichtbar gemacht, lädt zum Verweilen, hier funktional im Sinne des Arbeitens, ein. Dieses mobiliar-technische Arrangement legt eine spezifische Art der Arbeit nahe, d.h. sollte die Lehrperson etwas anderes als das durch den Tisch und die darauf befindlichen technischen Geräte Vorgegebene arbeiten wollen, muss sie den Platz wechseln. Der fixierte Tisch der Lehrperson gibt daher eher eine Benutzung vor, als dass er ein breites Potenzial hinsichtlich der Benutzbarkeit offeriert. Darüber hinaus befinden sich auf den beiden Tischen eine Kamera, verschiedene Kabel, Papiere, Stifte sowie ein Moderationskoffer, der aufgeklappt und somit zur schnellen Benutzung vorbereitet liegt - mitgebrachte mobile Objekte also, die aktiv so angeordnet worden sind. ${ }^{52}$

Im Raum befinden sich Stühle, die mit den Sitzflächen zum Tisch ausgerichtet an den Tischen stehen. Sie geben den eindeutigen Benutzbarkeitshinweis, dass und wo gesessen werden soll. Tische und Stühle zeichnen sich durch ein Ergänzungsverhältnis (Valenz) aus, sie treten zumeist in Kombination auf. ${ }^{53}$ Die Stühle sind genau wie die Tische mobil, sodass zugleich deren Umstellbarkeit und Nutzung beispielsweise für Gruppenaktivitäten offeriert wird. Die Stühle an den Tischen in der U-Form sind Holzstühle und sind alle gleich, während ein Stuhl anders aussieht: größer, gepolstert, höhenverstellbar und mit verstellbaren Armlehnen sowie einer ergonomischen Anmutung, er befindet sich am Tisch der Lehrperson. Tisch und Stuhl für die Lehrperson sind also in ihrer interaktionsarchitektonischen Gestaltung herausgehoben, sie sind es auch durch ihre Singularität. Die anderen, mehrfach vorkommenden Tische und Stühle sind alle gleich gestaltet, was damit interaktionsarchitektonischer Ausdruck des zahlenmäßigen sowie funktionsrollenbezogenen Verhältnisses zwischen Studierenden und Lehrperson ist.

Zur Möblierung kommt, neben der unbenutzten Tür stehend, ein Sideboard hinzu, auf dem ein Fernseher steht. Er steht mit der Bildschirmseite zur Wand und zeigt sich als dysfunktional, denn die Nutzung des Fernsehers wäre mit erheblichen Räumarbeiten verbunden. Neben dem Sideboard mit Fernseher steht ein beweglicher Overheadwagen mit Overheadprojektor. Auch er steht mit der zur Nutzung vorgesehenen Seite zur Wand, seine Benutzung wäre ebenfalls mit einigem Aufwand verbunden. Diese Aufstellungen im Raum fungieren als ein deutlicher Hinweis zur Nicht-Benutzung. ${ }^{54}$ Der Overheadwagen mit Overheadprojektor machen die Nutzung des Raums für Lehr-/Lerninteraktionen sichtbar, das gilt auch für das

52 Zum Umgang damit ausführlicher im Abschnitt Fallanalysen Beispiel 2: Die Lehrperson.

53 Hausendorf, Tische und Bänke (wie Anm. 7) 167.

54 Dafür spricht auch die Überholtheit dieser beiden technischen Geräte in moderner Hochschullehre, sie sind unüblich und nach institutionellen Vorgaben für die Lehre auch unerwünscht. Dennoch sind sie als Objekte Teil der relevanten Interaktionsarchitektur. 
frei stehende, beschreibbare Whiteboard sowie ein Flipchart. Das Flipchart befindet sich deutlich im Territorium der Lehrperson und macht damit Beschreibbarkeitsrechte sichtbar. Es ist von der Lehrperson weg gerichtet und damit lesbar für diejenigen, die an den Tischen sitzen, es zeigt über die gesamte Zeit der Videographie an diesem Tag einen handschriftlichen Guten-Morgen-Gruß. Durch die Schriftlichkeit in der multimodalen Interaktion wird die konditionelle Relevanz ${ }^{55}$ der Gruß- und Gegengrußsequenz verändert, sie bleibt aber ein Hinweis darauf, dass in diesem Raum derzeit soziale Interaktion stattfindet.

Ein Mülleimer, in der Ecke an der benutzten Tür stehend, und zwei Kübelpflanzen (von eher trauriger Gestalt), jeweils in den Ecken am Fenster, komplettieren die Möblierung des Raumes. Funktional lassen sie sich dem Aspekt der Verweilbarkeit zuordnen.

Die Flächen im Raum sind teils sichtbar markiert als funktionale Fläche für etwas. Es laufen Kabelkanäle auf einer Seitenwand entlang und an der Wand hinter der Lehrperson befindet sich eine fixierte Projektionsfläche. Ihre Funktionalität wird durch den an der Decke angebrachten Beamer und die Richtung, in die er zeigt, sichtbar gemacht. Zu den Benutzungshinweisen der Projektionsfläche gehört nicht nur die eindeutige technische Funktion, auch der Verweilbarkeit ist sie zuzuordnen. Beamer und Projektionsfläche schränken den Bereich der Begehbarkeit im Raum deutlich ein. Der Grund liegt darin, dass gewährleistet werden muss, dass es alle sehen können, und das wiederum stuft das zu Sehende in seiner Wichtigkeit sehr hoch. Die Flächen auf dem Boden werden nur durch Objekte sichtbar, sie markieren das Territorium der Studierenden und das der Lehrperson. Das persönliche Terrain der Lehrperson ist zwar nicht exklusiv für sie - hier befand sich beispielsweise der Redeort für die Studierenden, an dem sie das Auswertungsobjekt produzierten -, aber es ist bevorzugt und interaktiv unaufwändig von der Lehrperson begehbar. Das zeigt, dass Territorien nicht gleichermaßen und von allen begehbar sind. Vertrautheitsbasiert werden sie identifiziert, sie müssen jedoch auch verstanden werden, da sie über die Regelung ihrer Betretbarkeit ins Handeln eingreifen. In diesem Sinn sind sie »in einer stabilen Sozialtopografie des Klassenzimmers gewissermaßen kulturell verankert und besitzen dadurch eine gewisse Dauerhaftigkeit und Stabilität. $\ll^{56}$

55 Bei diesem typischen Merkmal gesprochener Sprache, den Paarsequenzen (neben GrußGegengruß auch z.B. Frage-Antwort), werden lokale Folgeerwartungen konstituiert, d.h. die erste Äußerunge schafft Bedingungen, die einen bestimmten Typ von Anschlusshandlungen erwartbar macht. Dieses Bedingungsgefüge wird als konditionelle Relevanz bezeichnet. Vgl. Deppermann, Gespräche (wie Anm. 12) 68.

56 Schmitt, Körperlich-räumliche Aspekte (wie Anm. 5) 20. Daran zeigt sich etwas für Klassenzimmer in Schule und Hochschule gleichermaßen Gültiges. Schmitt stellt seine Fallanalysen in einen »größtmöglichen Kontrast« (ebd., 17) und untersucht dazu die Ko- 
Gegenüber der Projektionsfläche befinden sich Fenster über die gesamte Breite des Raumes. Sie machen Außenwelt sichtbar, die für die Interaktion im Seminarraum nicht relevant ist, was sich auch an der Stellung der Tische und Stühle zeigt, die weitgehend von den Fenstern abgewandt sind. Allenfalls die Lehrperson könnte aus dem Fenster hinausschauen und die Außenwelt wahrnehmen.

Als Fazit kann zunächst die ausgeprägte Parzellierung des Raums festgehalten werden: Die Flächen sind funktional belegt, die Territorien sind markiert, es gibt wenig freien, ungenutzten Platz und es stehen viele Objekte herum, die Aufmerksamkeit auf sich ziehen können. Lediglich ein Objekt, der alte Fernseher, weist nur eine lose Verbindung als funktionaler Gegenstand für den Unterricht auf, alle anderen Objekte sind zweckdienlich (sogar die Pflanzen) im Hinblick auf das Lehren und Lernen sowie Gesprächeführen. Hinzu kommt, dass durch ein Überangebot an Tischen und Stühlen die Studierenden teils lose zu Gruppen verbunden, teils vereinzelt sitzen. Um in diesem Raum Lehr-/Lerninteraktionen herstellen zu können, greifen Interagierende auf institutionell geprägtes Raumnutzungswissen zurück, um nicht jedes Mal Interaktion im und genau in diesem Raum neu zu »erfinden«, sozialtopographisches Wissen erweist sich somit als »gesellschaftliches SpezialWissen $\ll .{ }^{57}$ In einem solchermaßen parzellierten Raum ist es eine pädagogische Herausforderung, zu zentrieren sowie einen Aufmerksamkeitsfokus zu schaffen und zu erhalten, anders als es in klassischen Hörsälen der Fall ist, in denen Lehrform ${ }^{58}$ und Raum stark korrelieren. Zugleich - und das ist der Sinn der Form Seminar muss die Lehrperson ein lebendiges Gespräch als zentrierte Interaktion initiieren sowie studentische Partizipation ermöglichen und leiten. Das hat für die Lehrperson in der Gestaltung der Interaktion Verknüpfungsleistungen zur Folge, wie sich im nächsten Beispiel zeigen wird.

\section{Fallanalysen Beispiel 2: Die Lehrperson}

Das nachfolgende Beispiel aus dem Seminar fügt dem Raum zunächst die Körper hinzu und zeigt, wie die Lehrperson als Fokusperson ${ }^{59}$ durch die Nutzung eines ges-

operation am Filmset und im Unterricht in der Schule. Nach Schmitt sind Unterricht bzw. das Klassenzimmer in Bezug auf die körperlich-räumlichen Aspekte ein vergleichsweise überschaubares Geschehen.

57 Hausendorf / Schmitt, Interaktionsarchitektur (wie Anm. 6) 16.

58 Vgl. die Ausführungen zu den Merkmalen von Vorlesung und Seminar im Abschnitt Handeln im gebauten Raum der Universität: Das Seminar.

59 Wenn von der Lehrperson im Interaktionsereignis gesprochen wird, wird der Terminus Fokusperson (wie Anm. 51) verwendet, um die Wahrnehmungsverhältnisse und die Interaktionskonstellation zu betonen. Wenn jedoch nur die institutionelle Rolle als Lehren- 
tischen Verweises auf ein technisches Objekt zum einen koordinativ und aufmerksamkeitszentrierend interaktiv handelt. Zum anderen stellt sie auf der Ebene des Sachbezugs globale Kohärenz der verschiedenen Hinsichten des gerade begonnenen Auswertungsgesprächs her.

Die Fokusperson eröffnet nach dem Anschauen der Videoaufnahme des Auswertungsobjekts das plenare Gruppengespräch mit einem Fragenbündel, in dem an eine offene Frage nach Rückmeldung drei spezifizierende, teils normaufrufende, Fragen angeschlossen werden: »okAY (-) was kÖnnen sie NAME sAgen; was ist ihnen Aufgefallen dabei; konnte man fOlgen, waren sie dabEI, «. ${ }^{60}$ Daraufhin beginnt der erste Student (=S1) mit einer Rückmeldung, die das erfragte Auffällige aufgreift (Zeile 06: mir ist nur grAde aufgefallen bei dem video dass (.) ähm du gerne U:nd sagst?//). Aufgefallen ist S1 also der einförmige Gebrauch des Konnektors und zur Kohärenzherstellung, S1 schließt eine Bewertung dessen an und verweist danach auf einen Unterschied in der eigenen Wahrnehmung, wonach ihm das im Vortrag kaum, im Video jedoch sehr stark aufgefallen sei. Die Studentin, deren Rede gerade ausgewertet wird (= AS), ${ }^{61}$ stimmt dieser Wahrnehmung zu, an dieser Stelle beginnt der Transkriptausschnitt:

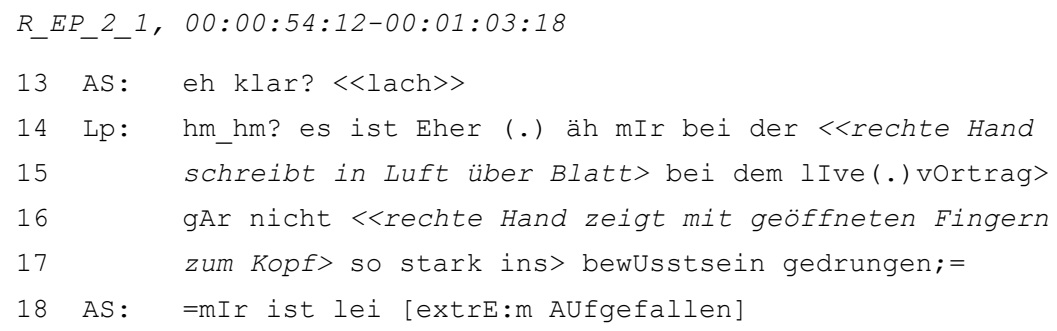

de gemeint ist, wird der Terminus Lehrperson verwendet. $\mathrm{Zu}$ den Interaktionsorganisationsaufgaben einer Lehrperson als Fokusperson in einer solchen Mehrpersoneninteraktion gehören auch die Organisation bzw. Zuteilung des Rederechts sowie die thematische Orientierung als Absicherung der Wissensvermittlung. Interaktionstypspezifische Aktivitäten von Auswertungsgesprächen kommen hinzu, das ist das Verhandeln von Aspekten der Wahrnehmung, der Beurteilung und der Intersubjektivierung eines Urteils. Eine interaktive Aufgabe für die Lehrperson besteht darin, diese Aspekte auf die zugrundeliegende Konvention oder fachlich relevante Wissensbestandteile hin zu befragen.

60 Transkriptionskonventionen vgl. Selting, GAT (wie Anm. 39).

61 AS kann wegen der Kameraperspektive im Bild nicht gesehen werden, da sie, von der Lehrperson aus gesehen, in der gegenüberliegenden linken Ecke des Raumes sitzt. 
Abbildung 2: Lehrperson als Fokusperson.

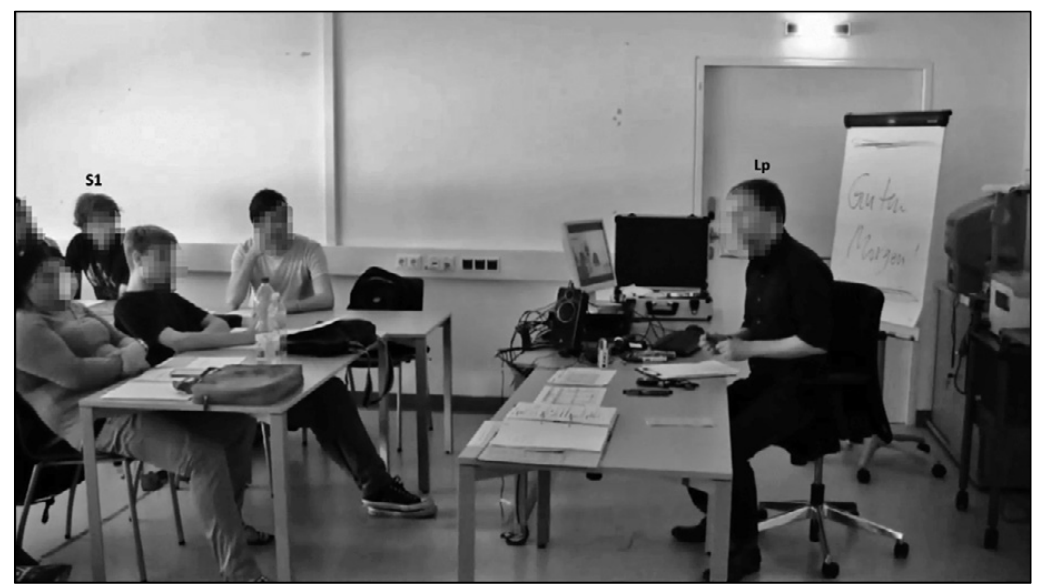

Quelle: K_Awg, R_EP_2_1, 00:01:03:18.

Um das Auswertungsgespräch als Gespräch sowie als Seminargespräch mit dem Ziel der Wissensvermittlung weiterzuführen, muss die Fokusperson auf den Beitrag von S1 sowie die Bestätigung durch AS reagieren. Dazu ratifiziert sie zunächst per $h m \_h m$ ? den Beitrag von S1 retrospektiv, markiert im Nachfolgenden jedoch Differenz, indem sie gerade nicht das Kritisierte, also den einförmigen Gebrauch des Konnektors und zur Kohärenzbildung, aufgreift. Vielmehr bearbeitet die Fokusperson den Aspekt der Differenz der Wahrnehmung in Abhängigkeit von der medialen Darbietung, indem sie die eigene Wahrnehmung anführt, wonach sie diese Differenz nicht feststellen konnte (Zeilen 14-17). AS insistiert durch erneute Zustimmung zur Rückmeldung von S1 (Zeile 18).

Während der Äußerung in den Zeilen 14-17 hält die Fokusperson, wie im Standbild ersichtlich, Blickkontakt zu AS und nicht zu S1, an dessen Beitrag sie anknüpft. Hier zeigen sich die Herausforderungen für die Koordination in einem Mehrpersonengespräch, denn der Blickkontakt erfordert eine Entscheidung. An dieser Stelle gäbe es dafür zwei Kandidat_innen: derjenige, auf dessen Beitrag geantwortet wird, sowie diejenige, deren Leistung ausgewertet wird. Die Haltung der Fokusperson im Bild besteht während der gesamten Äußerung, sie gibt den Rahmen für die beiden illustrierenden Gesten in den Zeilen 14 und 16 ab. Die Blickrichtung samt Kopfposition findet ihre Entsprechung in der Körperpositur ${ }^{62}$ als der Körper-

62 Die Kandidaten für die Analyse der körperlich-räumlichen Aspekte sind a) Körperliche Konstellationen, b) Körperpositur / Körperdrehung, c) Bewegung und Mobilität, d) Koordination und e) Gegenstände und Objekte. Die Auswahl ist keinesfalls erschöpfend. Vgl. Schmitt, Körperlich-räumliche Aspekte (wie Anm. 5) 32-42. 
drehung im Raum. Der Körper ist nicht gedreht, sondern mit durch die Arme geöffneter Körpervorderseite ausgerichtet auf AS. Die Studierenden, soweit sichtbar, schauen zur Fokusperson, sie sind (erwartbar) nicht in Bewegung, sondern sitzen; sie schreiben jedoch nicht mit. Letzteres zeigt sich häufig in den Daten, ist jedoch im Sinne des Qualifikationsziels des Seminars dysfunktional, da in den Auswertungsgesprächen reichlich fachbezogenes Wissen generiert und methodisch die germanistisch informierte Analyse von Texten bzw. Reden geschult wird.

Auf die erneute Zustimmung zur Rückmeldung von S1 durch AS (Zeile 18 im folgenden Transkript) folgt eine längere Sequenz der Fokusperson (Beginn in Zeile 19, die Äußerung geht weit über den präsentierten Ausschnitt hinaus), in der sie erklärt, wie diese Wahrnehmungsdifferenz zustande kommt. Den fachspezifischen Aspekt der Tauglichkeit verschiedener Konnektoren in Bezug auf die Kohärenzherstellung greift sie nicht auf. Interaktiv ist es für die Fokusperson erforderlich, eine detailliertere Erklärung zu geben, da ihre eigene Wahrnehmung als eine Expertenwahrnehmung (siehe Zeilen 14-17) argumentativ nicht ausreichend war.

Ausgehend von der Ruheposition, mit der die Fokusperson ihre Äußerung in Zeile 17 beendet und beibehält, ist in der ersten Äußerung (Zeile 19-20) der längeren Erklärsequenz folgende Bewegung zu sehen: Der Kopf wird weg von AS hin zum Bildschirm des Computers rechts auf dem Tisch gedreht, dabei zeigt die Hand des angewinkelten rechten Arms auf den Bildschirm. Die Finger sind zum Bildschirm hin geöffnet und mäßig gespannt, sie sind parallel mit dem Blick. ${ }^{63}$ Der Oberkörper dreht leicht mit und wird im oberen Bereich nach vorn rechts geschoben, er folgt also der Bewegung des Kopfes, und die Position des unteren Körpers bleibt unverändert. ${ }^{64}$ Die Bewegung des Körpers wird nach dem Kern der Gestenphrase in die Ruheposition zurückgeführt.

63 Die Geste hat formal und funktional starke Ähnlichkeit mit der von Stukenbrock so bezeichneten oHHv-Geste (= offene Hand Handfläche vertikal-Geste), deren Funktion zumeist das Präsentieren bzw. Anbieten von Objekten ist. Vgl. Stukenbrock, Deixis (wie Anm. 14) 149 ff. Grundsätzlich ist Gestik selbstverständlich weitaus präziser klassifizierbar (und analysierbar), als es hier vorgenommen wird, dies steht aber nicht im Fokus der Arbeit. Für einen Überblick vgl. Ulrike Bohle, Contemporary Classification Systems, in: Body - Language - Communication. An International Handbook on Multimodality in Human Interaction, hg. von Cornelia Müller et al. Bd. 2, Berlin 2014, 1453-1461 oder: Cornelia Müller, Redebegleitende Gesten: Kulturgeschichte, Theorie, Sprachvergleich, Berlin: 1998.

64 Diese Körperpositur wird als body torque beschrieben und meint: »different or diverging orientations of the body segments above and below two major points of articulation - the waist and the neck." Emanuel A. Schegloff, Body Torque, in: Social Research 65/3 (1998) 535-596, insb. 540. Die Funktion ist das Verbinden zweier Interaktionsbeteili- 


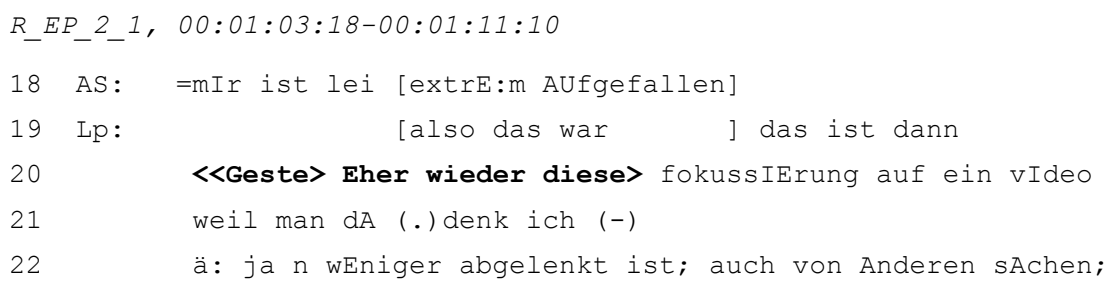

Abbildung 3: Geste der Fokusperson.

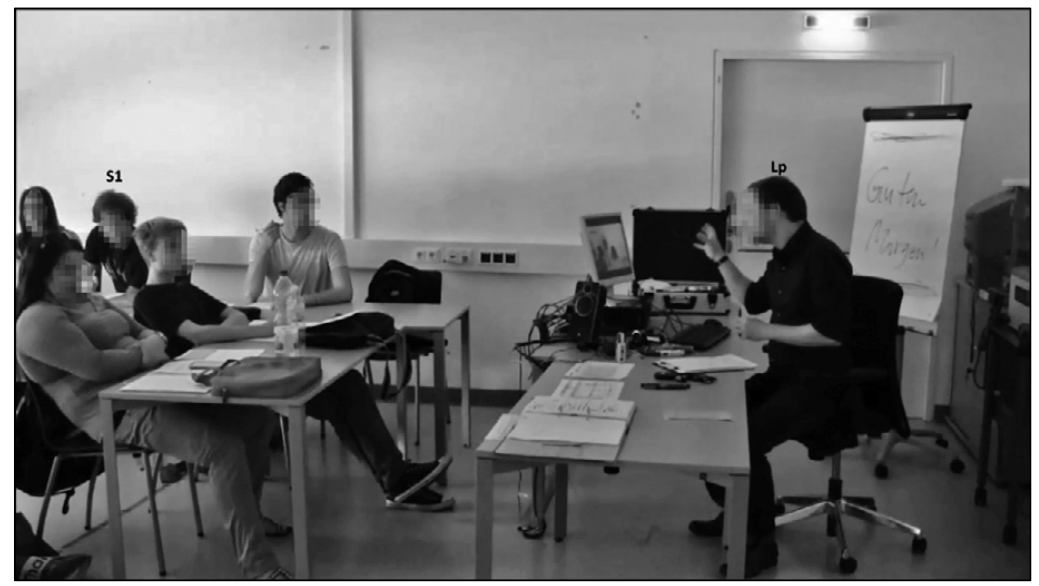

Quelle: K_Awg, R_EP_2_1, 00:01:04:24.

Die Bewegung erfolgt in Richtung des Bildschirms des Computers auf dem Tisch der Fokusperson, die Studierenden können jedoch den benutzbaren Teil, die Bildschirmvorderseite, nicht sehen. Durch diese Bewegung, die nicht kombiniert ist mit deiktischen Ausdrücken, aber verbunden mit Blickwechsel und gestischem Verweis, wird Koorientierung für die bzw. mit den Studierenden hergestellt und Aufmerksamkeit für das relevante Phänomen, das Video, etabliert. Damit die Geste als Erinnerungsaufruf an das Anschauen der Videoaufnahme im Wahrnehmungsraum der Studierenden eindeutig verortet ist, hätte die Fokusperson auf die - nun aber leere - Projektionsfläche zeigen müssen. So koorientiert die Fokusperson für sich, stellt fachliche, globale Kohärenz für das Seminar her und macht diese Kohärenzherstellung sichtbar. Diese Aufmerksamkeitslenkung ist nötig wegen der Differenz zwischen dem Beitrag von S1, auf den die Fokusperson Bezug nimmt, der sequenziellen Relevanz des Beitrags von AS sowie der eigenen thematischen Relevantset-

gungen, wobei eine als weiterhin bestehend gehalten wird - im Beispiel die Verbindung zu AS - und eine weitere temporär aufgemacht wird. 
zung. Interaktionsbezogen reagiert sein Handeln darauf, die thematische Differenz zu markieren. Dies gelingt dadurch, eine Verbindung von reden über Video und zeigen auf etwas Technisches in der eigenen Reichweite sowie zeigen auf etwas, das groß genug ist, um im Seminarraum von allen wahrnehmbar zu sein (diese Anforderung hätte bspw. die Kamera als Zeigekandidat nicht erfüllt), herzustellen. Die im Ausschnitt sichtbaren Studierenden machen die Koorientierung weitgehend mit, im Video zeigen sich kleine Mitbewegungen, es werden Köpfe gereckt, als gäbe es (auch für sie) etwas zu sehen.

Die Funktion dieser Körperbewegung, die in ihrer Flüchtigkeit und Kleinheit kaum bemerkbar ist, lässt sich in einer bestimmten Auffassung von Deixis als das »Sichtbarmachen des Sehens $«{ }^{65}$ bezeichnen, denn das tut die Fokusperson an dieser Stelle für alle Interaktionsteilnehmenden: Sie macht ihr eigenes Sehen und thematisches Fokussieren sichtbar, die Körperbewegung kann als den Wahrnehmungsraum strukturierende und damit Aufmerksamkeit bündelnde Geste gewertet werden. Aufmerksamkeitsbindung darf im Seminar nicht abreißen, dafür muss eindeutig sein, worauf sich die Aufmerksamkeit zu richten hat. Zugleich kann der Verweis auf das Objekt auch dazu dienen, mit der Flüchtigkeit mündlicher multimodaler Interaktion umzugehen und damit zum einen thematische Kohärenz herzustellen sowie zum anderen eine Verbindung zum Raum herzustellen, also zu situieren. Im Raum als Wahrnehmungsraum erfüllt die Fokusperson an dieser Stelle die interaktive Aufgabe, aus der Fülle des Wahrnehmbaren, wie im ersten Beispiel analysiert wurde, die für die laufende Interaktion relevanten Wahrnehmungen auszuwählen und interaktiv zu etablieren.

\section{Fallanalysen Beispiel 3: Die Studierenden}

Das folgende Beispiel aus demselben Auswertungsgespräch wenige Minuten später zeigt, wie interaktionsanforderungsbezogen Binnendifferenzierung in der Gruppe der Studierenden hergestellt wird, die sich in einem als Dyade gestalteten Interaktionsraum zeigt. Das dynamische Konzept des Interaktionsraums fokussiert die »räumlichen Arrangements der Körper der Interaktanten und ihre wechselseitige Ausrichtung und damit auf die Verfahren, mit denen sie sich im Hinblick auf ihr gemeinsames Handeln im Raum koordinieren. ${ }^{66}$ Die sprachlichen Handlungen sowie die Eigenheiten, die der Raum offeriert, können darin miteinander verknüpft werden.

65 Hausendorf, Interaktion im Raum (wie Anm. 13) 177.

66 Lorenza Mondada, Interaktionsraum und Koordinierung, in: Deppermann / Schmitt (wie Anm. 4) 55-94, insb. 55. Zu Interaktionsdyaden vgl. auch Schmitt, Körperlich-räumliche Aspekte (wie Anm. 5) 54 ff. 


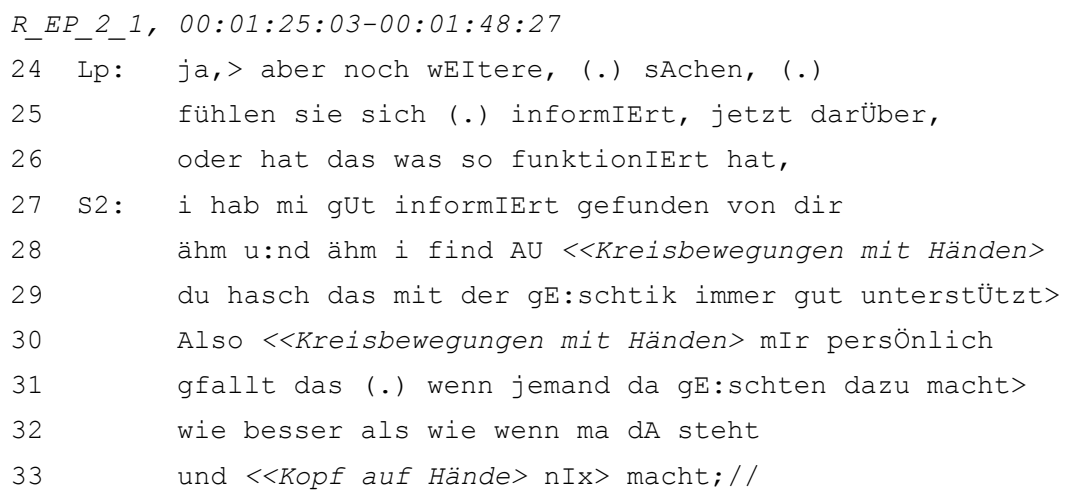

Abbildung 4: Interaktionsdyade.

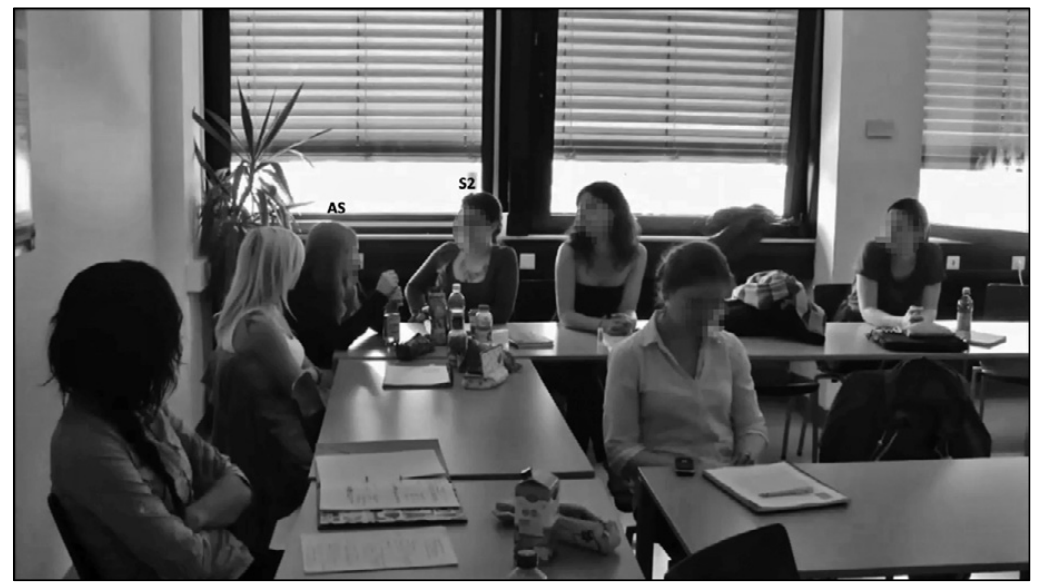

Quelle: K_Awg, R_EP_2_1, 00:01:25:03.

Eine der interaktiven Anforderungen, vor denen die Studierenden in einem plenaren Auswertungsgespräch stehen, ist die aus dem Mehrpersonengespräch sowie den Spezifika des Seminars erwachsende Mehrfachadressierung der Beiträge. Für die Interagierenden heißt diese Adressierungsaufgabe, dass sie deutlich machen müssen, wer (zunächst) angesprochen ist und diese Adressierung muss sowohl für den/die Student_in, dessen/deren Leistung ausgewertet wird, als auch für die anderen Studierenden sowie für die Lehrperson klar erkennbar sein. In Bezug auf das Gespräch als Seminargespräch mit Bildungszielen kommt hinzu, dass etwas inhaltlich-fachbezogen Relevantes gesagt werden muss, was von der Lehrperson, wie in unterrichtlicher Interaktion üblich, gegebenenfalls evaluiert, ergänzt oder richtiggestellt wird. In diesem Beispiel löst die Studentin (= S2) das Adressierungsproblem 
in erster Linie räumlich-körperlich, indem sie eine Interaktionsdyade mit der Studentin, deren Arbeit gerade ausgewertet wird (AS), vor den anderen etabliert.

Nachdem die Lehrperson (Zeilen 24-26) einen neuen Impuls zu Weiterführung der Auswertung gibt und explizit dazu einlädt (ja,> aber noch wEItere, (.) sAchen,), schlägt sich S2 per Handzeichen vor und bekommt durch eine auf sie weisende Geste das Rederecht erteilt. S2 fängt sofort an zu reden und startet die Rückmeldung ohne Hesitationen. Sie bezieht sich darin thematisch präzise auf die zweite Frage (Zeile 25: fühlen sie sich (.) informIErt, jetzt darÜber,), indem sie die Formulierung aufgreift und die Frage bejahend beantwortet (Zeile 27), sie reagiert also auf die konditionelle Relevanz des Frage-Antwort-Schemas. Danach schließt sie eine Begründung dafür an, bewertet diese Handlungsweise als positiv (Zeile 29: $d u$ hasch das mit der gE:schtik immer gut unterstÜtzt) und markiert diese positive Bewertung explizit als eine persönliche Einschätzung und spezifiziert, was ihr in Bezug auf den Gegenstand redebegleitende Gestik nun genau gefalle.

Die von S2 initiierte Interaktionsdyade wird durch die zueinander positionierten Oberkörper von AS und S2 konstelliert, sie nutzen dafür ihre räumlich nahe Sitzposition nebeneinander über Eck. Die Dyade zeichnet sich in der Körperpositur durch die symmetrische Drehung und Ausrichtung der Oberkörper sowie der beiden Köpfe zueinander aus, sodass der Blickkontakt auf einer Linie von beiden gehalten werden kann. AS und S2 sind koorientiert und koordiniert aufeinander, kooperierend im Zuhören bzw. Geben einer persönlichen Rückmeldung.

Während AS nahezu unbeweglich zuhört und Blickkontakt hält, zeichnet sich das Handeln von S2 durch vermehrte Bewegung aus. Zum einen sind es Gesten in den Zeilen 28-29 und 30-31. An diesen Stellen vollführt S2 Bewegungen beider Hände, die in Brusthöhe mit offenen Handflächen und als iterative, parallel ausgeführte, einen Kreis von unten am Körper nach oben und vom Körper weg nach außen zeichnende Handbewegungen durchgeführt werden. Zum anderen ist das die Position des Oberkörpers. Die Zuwendung von S2 zu AS durch die Drehung des Oberkörpers wird durch das Aufstützen der rechten Hand auf dem rechten Bein unterstützt und unterstrichen, der linke Arm liegt auf dem Tisch, dreht aber in die gleiche Richtung mit. ${ }^{67}$ Der Oberkörper ist leicht zurückgelehnt, Kopf und Nacken sind in einer Linie dazu. Somit ist der Beitrag (Zeile 27-33) körperlich-räumlich klar an AS adressiert.

$67 \mathrm{Ob}$ auch hier ein body torque (wie Anm. 64) vorliegt, kann aus dem Video nicht hinreichend sicher erschlossen werden, es wäre jedoch funktional naheliegend. 
Abbildung 5: Ruheposition nach der Interaktionsdyade.

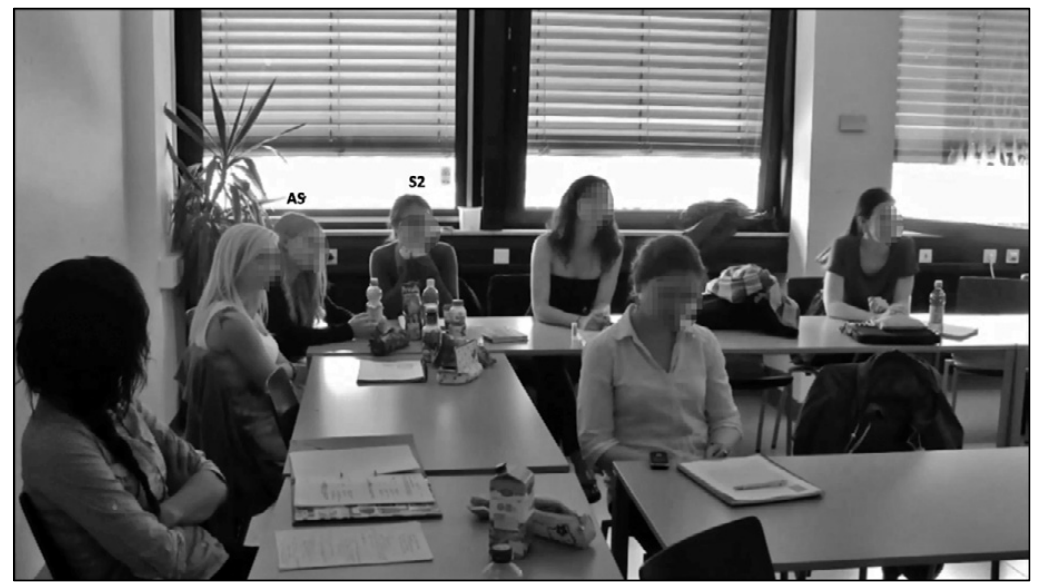

Quelle: K_Awg, R_EP_2_1, 00:01:48:27.

Eine Entsprechung der körperlich-räumlich hergestellten Interaktionsdyade auf der prosodisch-stimmlichen Ebene ist die für ein universitäres Seminar als relativ stark zu bezeichnende Dialektverwendung von S2, die im Vergleich zu ihren anderen Redebeiträgen in diesem gesamten Auswertungsgespräch klar markiert ist. Die nähesprachliche, Distanz reduzierende Dialektverwendung ist funktional für das Herstellen der Dyade. Zugleich zeigt jedoch der gesamte Beitrag von S2 auf der prosodischen Ebene Merkmale der Adressierung gerade nicht für die räumliche Nähe, sondern für die Distanz: er ist ausreichend laut, schnell, mit hoher Artikulationspräzision und Artikulationsspannung gesprochen. All das sind formale Merkmale der Adressierung des Beitrags an alle, er ist gesprochen für einen großen Raum, in dem alle verstehen können müssen. Damit reagiert S2 zum einen auf die Sitzposition über Eck, zugleich schafft sie durch das Zurücklehnen ausreichend Distanz, um nicht in sogenanntes privates Sprechen zu fallen, sondern so, dass es für alle anderen wahrnehmbar bleibt. Das funktioniert, denn die anderen Studierenden sind per Blick auf diese Dyade gerichtet. Auch die Blickrichtung von S2 entspricht der primären Adressierung an AS, der Blick zu AS besteht durchgängig, mit Ausnahme der Hesitationspartikel (ähm u:nd ähm) in Zeile 28 sowie in Zeile 31 (auf jemand), da erfolgt ein Blick zur Lehrperson. Die Veränderungen im Blickverhalten zeigen die funktionale Bewältigung der Mehrfachadressierung.

S2 beendet die Dyade während der Äußerung in Zeile 33 (und $<<$ Kopf auf Hände $>n I x>$ macht;//), dabei geht der Blick weg von AS, der Oberkörper dreht in die durch Tisch/Stuhl und U-Form nahegelegte Ausrichtung zurück, die Arme werden angewinkelt auf den Tisch gestützt und der Kopf wird mit kurzer, nachdrückli- 
cher Bewegung genau auf der Akzentuierung von $n I x$ auf den verschränkten Händen abgelegt. AS bleibt zunächst in der dyadischen Position und dreht erst mit Beginn der nächsten Äußerung der Lehrperson zurück in die plenare Anordnung. AS und S2 sind koordiniert beim Beginn der Dyade, sie sind es aber nicht bei deren Beendigung, also der Rückführung in die körperlich-räumliche Ruheposition des plenaren Auswertungsgesprächs (Ruheposition: siehe Abbildung 5). Beendet ist das Thema der Angemessenheit von redebegleitender Gestik mit dem präsentierten Ausschnitt keineswegs, es folgen mehrere Minuten Gespräch darüber mit der gesamten Gruppe.

Die Interaktionsdyade ist funktional für diese Rückmeldesequenz und ermöglicht S2 eine deutliche Adressierung ihres Beitrags. So wird auf die interaktive Anforderung im Auswertungsgespräch, demjenigen, dessen Arbeit ausgewertet wird, eine persönliche, konkrete und relevante Rückmeldung zu geben, reagiert und zugleich angezeigt, dass der eigene Beitrag sachbezogen für das Seminar relevant und also verallgemeinerbar ist. Die Herstellung einer Dyade, wie im präsentierten Beispiel, ist eine gemeinsame, interaktionsbezogene Antwort auf eine interaktionsbezogene Anforderung und somit ein dynamisches, darauf zugeschnittenes Geschehen. Die Interagierenden greifen also zur Herstellung von Interaktionsraum auf den ausgestatteten Raum zurück.

\section{FAZIT}

In den Fallanalysen wurde der Fokus im ersten Schritt auf den Raum und dessen Potenziale für die Interaktion gelegt, im zweiten Schritt auf die Interaktionsbeteiligten des Seminars, Lehrperson und Studierende. Der Grund für diese Auswahl liegt in der vielfach angeführten wechselseitigen Bezogenheit von Körper und Raum, wonach erst der Körper Raumerfahrung möglich macht. Es wurde das interaktive Handeln der Interaktionsbeteiligten in Bezug auf den Raum als Ressource, die Art des Zusammenhangs der Lösung interaktiver Aufgaben durch relevant gesetzte Aspekte von Raum analysiert und der Zusammenhang zwischen körperlichen und räumlichen Konstellationen in diesem speziellen Interaktionstyp aufgezeigt.

Der Interaktionstyp Auswertungsgespräch zeigt sich als integraler Bestandteil des übergeordneten Lehrveranstaltungstyps universitäres Seminar, wobei die spezifischen Eigenschaften der Daten als Lehrveranstaltungstyp Seminar in der tertiären Bildung herausgestellt werden müssen. Das Merkmal der Lehr-/Lerninteraktion teilen die Daten zwar mit schulischer Interaktion, aber universitäre Interaktion im Seminar unterliegt anderen institutionstypischen, kontextualisierenden Besonderheiten und Einflussfaktoren. Es zeigt sich, dass Raumnutzung und die körperlichen Konst- 
ellationen fachspezifisch sind bzw. auf fachspezifische Anforderungen reagieren. ${ }^{68}$ Welche Merkmale für ein germanistisches Seminar mit dem Schwerpunkt Rhetorik gelten, ist noch nicht abschließend geklärt. Bislang ist jedoch deutlich, dass das Vorliegen eines Auswertungsobjekts, unabhängig von der medialen Darbietungsweise als ein Text oder als Videoaufzeichung einer Rede, fachspezifische Interaktionsaufgaben stellt, die spezifische räumlich-körperliche Konsequenzen haben.

Die - selbstverständlich in verschiedenen Hinsichten verfeinerbaren - Analysen von Mikrophänomenen der Interaktionen zeigen die Alltäglichkeit des Lehrens und Lernens, sie zeigen, wie unproblematisch solche Situierungen der Interaktion sein können. Das erste Beispiel konzentrierte sich auf den gebauten und gestalteten Raum sowie auf das, was durch die Architektur in Bezug auf die Interaktionskonstitution erwartbar gemacht wird und welche Ergebnisse die Perspektiven der Sichtbarkeit, Hörbarkeit, Begehbarkeit, Begreifbarkeit und Verweilbarkeit auf die räumlichen Arrangements erbringen. An diesem Beispiel zeigt sich zugleich, wie architektonische Erscheinungsformen Lösungen für interaktive Probleme der Lehr/Lerninteraktion darstellen, beispielsweise die Platzierung der Projektionsfläche als gut sichtbar oder die Anordnung von Tischen und Stühlen, um plenare Gespräche einer gesamten Gruppe effektiv zu ermöglichen.

In den weiteren Beispielen stand die stattgehabte Interaktion im Mittelpunkt. Im zweiten Beispiel, das den Fokus auf die Lehrperson und deren Handeln legt, wurde gezeigt, wie die Lehrperson im kleinräumig parzellierten Raum aus dem Angebot an technischen Objekten gestisch auswählt, in die laufende Interaktion eingliedert und damit der interaktiven Aufgabe der Koorientierung der Interagierenden sowie der thematischen Kohärenzherstellung für das Seminar nachkommt. Im dritten Beispiel wurde gezeigt, wie durch das Herstellen einer Interaktionsdyade innerhalb der Gruppe der Studierenden Raum als Interaktionsraum konstituiert wird. Diese Interaktionsdyade reagiert auf die interaktionsspezifische Notwendigkeit des zwar personenspezifischen, aber zugleich für das Seminar relevanten Rückmeldens; die kurze, vorübergehende und damit funktionale körperlich-räumliche Konstellation löst das Adressierungsproblem. Die im Aufsatz präsentierten Beispiele sind in erster Linie aussagekräftige Sequenzen, sie zeigen häufiges, deutliches sowie typisches Handeln, woraus sich Regularitäten ableiten lassen. Es zeigt sich, dass die für die Interaktion notwendige Koorientierung, Koordination und Kooperation systematisch durch die Gestaltung des Raumes nahegelegt werden sowie durch die Ver-

68 Beispielsweise nennt Putzier als Charakteristikum von Chemieunterricht, das etwas gemacht bzw. etwas vorgemacht werden muss und auf diese Weise ein Demonstrationsraum eröffnet wird, mit dem der Raum um den Versuchstisch sowie die Durchführung des Versuchs gemeint ist. Vgl. Eva-Maria Putzier, Der >Demonstrationsraum< als Form der Wahrnehmungsstrukturierung, in: Raum als interaktive Ressource, hg. von Hausendorf / Mondada / Schmitt (wie Anm. 3) 275-315. 
trautheit bzw. Konventionalisiertheit der gebauten Räume der Universität, genauer des Seminarraums.

Interagierende gehen mit dem sie umgebenden gebauten Raum um, dabei greifen sie auf auch institutionell geprägtes Raumnutzungswissen zurück. Das ist für Daten aus der tertiären Bildung wichtig, zeigt sich doch, dass Studierende eine eigene Bildungsinstitutionenbiografie haben und diese, trotz der Novität in ihrem eigenen Handeln und aller Eigenheiten der Universität, produktiv als Ressource nutzen können. Eine institutionsbezogene praktische Konsequenz aus solchen Analysen ließe sich an dieser Stelle andeuten: Danach sollte die Institution Universität großes Interesse daran haben, das von ihr als wesentlich erachtete Seminar - eben weil es die Lehrform ist, die forschenden Habitus ausbildet - auch durch den gebauten Raum zu unterstützen, also Bedingungen zu schaffen, in denen solche Lehre optimal ermöglicht wird. 